\title{
PSICOFÁRMACOS NO TRATAMENTO DA DEPENDÊNCIA QUÍMICA: UMA REVISÃO
}

\author{
PSYCHOPHARMACY IN THE TREATMENT OF CHEMICAL DEPENDENCE: A REVIEW
}

Marcos Fernando Knevitz ${ }^{1}$ Danieli Fernanda Buccini²

Recebido em: 21 mar. 2017

Aceito em: 01 abr. 2018

RESUMO: A farmacoterapia atua no tratamento da dependência química, complementando outras atividades que procuram melhorar os aspectos da vida dos pacientes. Os tratamentos farmacológicos têm por finalidade prevenir ou atenuar a síndrome de abstinência, diminuir a fissura e atuar no tratamento das comorbidades. O objetivo deste trabalho foi revisar o modo de atuação dos psicofármacos no tratamento das dependências, além de verificar sua relação com as principais classes de substâncias de abuso. A metodologia utilizada foi a revisão sistemática, utilizando como critérios de avaliação a população envolvida de adultos e adolescentes, a intervenção referindo o uso de psicofármacos no tratamento da dependência química e como desfecho os efeitos dos medicamentos nos indivíduos. Apesar de apresentarem bons resultados no tratamento da dependência de álcool, nicotina e opioides, importantes adições como maconha, cocaína e seus derivados ainda não possuem tratamentos farmacológicos com evidências positivas comprovadas que permitam sua utilização. Desse modo, são necessárias mais pesquisas para a busca de novos medicamentos que auxiliem o tratamento dessas dependências, além da participação ativa do paciente no processo.

Palavras-chave: Psicofármacos. Tratamento. Dependência Química. Comorbidades. Fissura.

ABSTRACT: Pharmacotherapy works in the treatment of chemical dependency, complementing other activities which seek to improve the quality of patients' lives. Pharmaceutical treatments aim to prevent or attenuate a withdrawal syndrome, reduce craving and act in the treatment of comorbidities. The objective of this study is to review the way psychoactive drugs work in dependence treatment, as well as to verify their relationships with the major classes of substances of abuse. The methodology used was the systematic review, using as evaluation criteria the population of adults and teenagers engaged, the intervention related to the use of psychotropic drugs in the treatment of chemical dependence and as an outcome the effects of the drugs in the individuals. Despite the fact that it presents good results in treatment of alcohol dependence, nicotine and opioids, important additions such as marijuana, cocaine and its derivatives are not pharmacological treatments with proven positive evidence which allow its use yet. In this way, further studies are needed for the searching of new drugs for the treatment of addictions, besides the active participation of the patient in the process.

Keywords: Psychopharmaceuticals. Treatment. Chemical Dependency. Comorbidities. Craving.

\footnotetext{
1 Farmacêutico pela Universidade Luterana do Brasil (ULBRA). Mestre em Saúde Coletiva (ULBRA). marcosknevitz@hotmail.com.

2 Bióloga pela Universidade Anhanguera (UNIDERP) Mestre em Biotecnologia pela Universidade Católica Dom Bosco (UCDB). dfbuccini@gmail.com.
} 


\section{INTRODUÇÃO}

Dados recentes acerca do uso mundial de drogas ilícitas mostram que no ano de 2013 o número de usuários chegou a um total de 246 milhões e estima-se que uma entre vinte pessoas, com idade entre 15 e 64 anos, tenha utilizado alguma substância psicoativa (SPA) ilícita no último ano. A magnitude da questão se torna mais evidente quando se considera que mais do que um em dez consumidores de drogas é um usuário problemático, ou seja, apresenta algum tipo de distúrbio devido a este uso. Além disso, apenas um a cada seis desses consumidores problemáticos tem acesso a algum tipo de tratamento (UNODC, 2015).

A consequência deste uso abusivo é o aumento na carga global de doença e anos de vida produtiva perdidos (DEGENHARDT, et al, 2010), além de um número inaceitável de mortes relacionadas com drogas: cerca de 187100 pessoas perderam a vida prematuramente em 2013 (UNODC, 2015).

Apesar da utilização de alguma SPA não indicar necessariamente ser uso abusivo, ou mesmo de estado de dependência, os dados epidemiológicos demonstram que o grande volume de uso destas substâncias pela sociedade leva a necessidade de uma maior atenção com a dependência química (DQ).

As diferentes dimensões envolvidas no processo da dependência, além do uso prolongado das SPA, como os aspectos sociais, culturais, educacionais e comportamentais influenciam no tratamento desta síndrome (FIGLIE, BORDIN e LARANJEIRA, 2010). Desse modo, o desenvolvimento de tratamentos farmacológicos efetivos para os quadros de dependência tem sido prejudicado pela ainda deficiente compreensão das alterações bioquímicas que as SPA promovem no cérebro humano, assim como a relação entre essas alterações cerebrais e as modificações comportamentais, presentes no processo (VOLKOW, FOWLER e WANG, 2003).

Nesse contexto, as pesquisas têm procurado esclarecer as bases neurobiológicas do processo de dependência, pelo melhor entendimento dos mecanismos cerebrais envolvidos e nesse contexto, o sistema dopaminérgico tem se apresentado como potencialmente envolvido em mecanismos que desempenhariam papel destacado nos processos de dependência e abstinência (VOLKOW, FOWLER e WANG, 2003).

A farmacoterapia tem papel importante no tratamento da dependência química, complementando outras atividades que procuram melhorar os aspectos da vida dos pacientes, através da equipe multidisciplinar. Desse modo, os tratamentos farmacológicos teriam por finalidade prevenir ou atenuar a síndrome de abstinência, diminuir a fissura e atuar no tratamento das comorbidades (FONSECA e LEMOS, 2011).

Sendo assim, o objetivo deste trabalho é verificar o modo de atuação dos fármacos no tratamento da $D Q$, além de examinar sua relação com as principais classes de SPA. 


\section{METODOLOGIA}

A busca pelas referências foi realizada através do portal da CAPES - Coordenação de Aperfeiçoamento de Pessoal de Nível Superior nas plataformas de pesquisa do próprio portal, BVS - Biblioteca Virtual em Saúde e EBSCO - Elton B. Stephens Company, utilizando as informações contidas nas diversas bases de dados eletrônicas, principalmente: MEDLINE - Medical Literature Analysis and Retrieval System Online, LILACS - Literatura Latino-Americana e do Caribe em Ciências da Saúde e SCIELO - Scientific Electronic Library Online. As palavras chave foram convertidas em descritores obtidos no site Ciências da Saúde (http://decs.bvs.br), sendo, em português: psicotrópicos; tratamento do abuso de substâncias; transtornos relacionados ao uso de substâncias; comorbidades; fissura. Em inglês: psychotropic drugs; substance abuse treatment; substance related disorders; comorbidities; craving. Os dados foram coletados entre julho e setembro de 2016, utilizando amostragem por conveniência, sendo selecionados os estudos identificados conforme a abrangência temporal entre 2006 e 2016 e quando o idioma utilizado era português, inglês ou espanhol. Além disso, foram utilizados como critérios para avaliação a população envolvida (adultos e adolescentes), a intervenção (uso de psicofármacos no tratamento da DQ) e o desfecho (efeitos dos medicamentos nos indivíduos).

A análise do material coletado foi realizada inicialmente pela leitura do título, resumo e palavras-chave, onde os artigos que estavam alinhados com os objetivos da pesquisa e atenderam os critérios de inclusão foram selecionados. Após foi feita a leitura da introdução e conclusão dos artigos e aqueles que não atenderam os objetivos da busca e os critérios de inclusão foram eliminados. Finalmente os artigos selecionados foram submetidos à leitura completa, focando nos objetivos e critérios de inclusão. Os artigos que passaram por este filtro foram considerados relevantes para a pesquisa e fazendo parte da revisão.

\section{RESULTADOS E DISCUSSÃO}

Os resultados da busca encontraram 288 artigos que atendiam os critérios de inclusão, sendo selecionados 49 destes trabalhos que se aproximaram dos objetivos da pesquisa e foram lidos completamente. Além disso, foram buscados 10 trabalhos encontrados nas referências dos artigos lidos e que entraram na revisão devido à especificidade do assunto. Os achados dessa revisão estão divididos em duas partes: 0 modo como os medicamentos atuam nos processos de tratamento da dependência química e a relação entre a psicofarmacoterapia e as principais classes de substâncias de abuso.

\section{MODO DE ATUAÇÃO DOS FÁRMACOS NOS PROCESSOS DE DEPENDÊNCIA}


QUÍMICA

A abordagem farmacológica tradicional do tratamento da DQ busca interferir nos processos neurológicos afetados pelo abuso das substâncias psicoativas. Esses procedimentos podem envolver o reforço positivo ao efeito das substâncias de abuso por buscar o bloqueio ou a redução da sensação de prazer das drogas ou pelo reforço negativo que visa aliviar as condições de abstinência (FURST, RIBA, AL-KHRASANI, 2013). Além disso, outras abordagens farmacológicas têm como alvo as vulnerabilidades individuais do dependente, como déficit cognitivo, diferenças de sexo e comorbidades psiquiátricas (FORRAY e SOFUOGLU, 2012).

\section{FÁRMACOS QUE ATUAM NO REFORÇO POSITIVO}

O reforço positivo pode ser definido como qualquer estímulo que aumente a probabilidade de volta a um comportamento anterior, normalmente envolvendo uma recompensa hedônica. A autoadministração é a principal medida para o reforço das SPA de abuso e em quase todas as drogas, se traduz na recompensa subjetiva ou o "gostar" em humanos (BERRIDGE, ROBINSON, ALDRIDGE, 2009). Apesar do papel da dopamina no comportamento aditivo ainda continuar a ser discutido, este neurotransmissor é considerado como de fundamental relevância no processo de recompensa cerebral (DALLEY e EVERITT, 2009), embora este sistema também seja mediado por outros neurotransmissores, incluindo opioides endógenos, ácido g-aminobutírico (GABA) e endocanabinoides (HORDER, 2010). A identificação dos mecanismos destes neurotransmissores que participam da mediação dos aspectos da DQ a nível cerebral pode facilitar o desenvolvimento de novos alvos para a farmacoterapia das desordens causadas pelo uso abusivo de drogas.

$\mathrm{Na}$ abordagem agonista do reforço positivo os fármacos atuam nos mesmos receptores de neurotransmissores que são estimulados pelas SPA de abuso e sua principal estratégia é substituir as drogas de abuso, que possuem ação mais curta por fármacos de ação mais longa e segura, com possibilidade de posologia controlada. Exemplos tradicionais de tratamentos agonistas incluem metadona para a dependência de opioides e tratamento de reposição de nicotina para tabagistas. Outro modo de atuação dos tratamentos agonistas é pelo aumento da dopamina extracelular, pois além do seu papel no reforço ao uso de drogas, o déficit deste neurotransmissor resulta na manutenção do uso crônico de SPA (FORRAY e SOFUOGLU, 2012). Como exemplos, a dextroanfetamina, que aumenta a liberação de dopamina sináptica por interromper o armazenamento de dopamina em vesículas intracelulares (LONGO, 2010) e o metilfenidato que, como a cocaína, aumenta os níveis de dopamina sináptica por inibição da recaptação de transportador de monoaminas (LEVIN, 2007), têm sido testados no tratamento da dependência de cocaína e metanfetamina.

O transporte das moléculas da dopamina pelos circuitos cerebrais também estão 
sendo alvo de estudo e fármacos como a lisdexanfetamina (JASINSKI e KRISHNAN, 2009) e o modafinil, que são estimulantes que estão sendo testados na adição por cocaína e metanfetamina, atuam como fracos transportadores da dopamina, o que aumenta a concentração desse neurotransmissor a nível sináptico. O modafinil, além de aumentar os níveis de dopamina sináptica, também estimula os neurônios hipotalâmicos de orexina, reduz a liberação de GABA e aumenta a liberação de glutamato (MARTINEZ-RAGA, KNECHT, CEPEDA, 2008).

Outras drogas que aumentam a dopamina foram avaliadas como potenciais intervenções terapêuticas com resultados mistos. A bupropiona, que atua como um inibidor da recaptação da dopamina e é utilizada no tratamento do tabagismo e a norepinefrina que produz aumento extracelular dos níveis de dopamina e tem sido usada com bons resultados na dependência do álcool, não demonstraram efeitos significativos para a cocaína e metanfetamina (SHOPTAW, 2008). Já o dissulfiram e o nepicastat são fármacos de abordagem agonista devido à sua função como um inibidor da dopamina-b-hidroxilase, o que proporciona um aumento dos níveis de dopamina. O dissulfiram já é utilizado com efetividade no tratamento do alcoolismo, mas está sendo testado, assim como o nepicastat, devido ao seu mecanismo de ação, para o tratamento da dependência de cocaína (HEIDBREDER e NEWMAN, 2010).

De outra forma, na abordagem antagonista do reforço positivo, apesar dos fármacos também agirem nos mesmos sistemas de neurotransmissores das SPA de abuso, agora atuam no bloqueio dos efeitos dessas substâncias. Exemplos de antagonistas incluem a naltrexona e a buprenorfina para o tratamento da dependência de opioides, sendo a naltrexona também utilizada para a dependência de álcool, e a vareniclina para a dependência da nicotina. Já o nalmefeno, que possui mecanismo de ação semelhante a naltrexona, ainda está sendo testado no tratamento do alcoolismo (FORRAY e SOFUOGLU, 2012).

Antagonistas e agonistas parciais dos receptores de dopamina D3 são alvos promissores para pesquisa no tratamento da dependência de cocaína, pois enquanto os agonistas D3 aumentam os efeitos de recompensa da cocaína, antagonistas D3 ou agonistas parciais atenuariam o reforço da cocaína e reduziriam a autoestimulação e reposição de cocaína e anfetaminas (PENG, 2009). A eficácia dos agonistas parciais D3 e antagonistas ainda precisa ser analisada em estudos humanos.

\section{FÁRMACOS QUE ATUAM NO REFORÇO NEGATIVO}

Os efeitos reforçadores negativos da abstinência causada pela utilização de substâncias de abuso estariam associados a alterações neuroadaptativas em vários sistemas de neurotransmissores cerebrais, como dopamina, norepinefrina, fator liberador de corticotropina (CRF), GABA e glutamato. Estas mudanças neuroadaptativas afetariam o sistema de recompensa cerebral causando os sintomas clínicos de abstinência, como 
fissura e estados negativos de humor. O aumento da atividade da norepinefrina e do CRF está associado com o estado de ansiedade observado durante a abstinência aguda, enquanto que as alterações nos sistemas de dopamina, GABA e glutamato estão relacionados com o quadro de fissura (KOOB e VOLKOW, 2010).

A redução dos níveis dos neurotransmissores que afetam esse circuito de recompensa cerebral é presumida como pressuposto para mediar os sintomas clínicos verificados e a anedonia observada nos estados de abstinência de drogas (SCHMIDT e PIERCE, 2010). São exemplos de fármacos que atuam no reforço negativo de drogas a antalarmina, que é um antagonista seletivo de ação central dos receptores da CRF a metadona e a buprenorfina, que aliviam sintomas de abstinência de opioides, a bupropiona e a vareniclina, que reduzem os sintomas de abstinência da nicotina e atenuam estados de humor negativos após cessação de fumar (FORRAY e SOFUOGLU, 2012).

Outros compostos que atuam no reforço negativo são os que têm como alvo o sistema da orexina, que esta sendo relacionado aos sintomas de fissura e recidivas do uso de SPA de abuso. O exemplo dessa classe é o suvorexanto, um antagonista da orexina, desenvolvido inicialmente para insônia (KHOO e BROWN, 2014).

Medicamentos que tem seu foco sistema noradrenérgico também estão demonstrando resultados promissores para tratamentos do uso abusivo de SPA. O fármaco lofexina, agonista alfa adrenérgico, tem seus efeitos estudados em usuários de cocaína e opioides. Ensaios clínicos estão em andamento para testar a eficácia do carvedilol, um antagonista alfa e beta adrenérgico e da guanfacina, uma agonista alfa adrenérgica, para a dependência de psicoestimulantes. A prazosina, um antagonista alfa adrenérgico, demonstrou em ensaios pré-clínicos que pode diminuir a autoadministração de etanol e heroína e a guanfacina está sendo testada na fissura por cocaína, com resultados promissores (FORRAY e SOFUOGLU, 2012).

Potenciais terapias farmacológicas para o tratamentos dos distúrbios causados pelo uso abusivo de SPA, possuem como base o estudo do papel do glutamato e do GABA nos processos da dependência. A memantina e a acetilcisteína tem como alvo o sistema glutamato e o baclofeno e a vigabatrina atuam no GABA (FORRAY e SOFUOGLU, 2012). Apesar dessa possibilidade, os ensaios com estas linhas de pesquisa ainda estão incipientes.

\section{FÁRMACOS QUE ATUAM NAS COMORBIDADES}

Os distúrbios causados pelo uso abusivo de substâncias e outros transtornos psiquiátricos tem uma estimativa de ocorrência simultânea com prevalência superior a $60 \%$ (BRADY, 2007), sinalizando a necessidade de atenção especial com essa associação durante as intervenções terapêuticas. Além disso, existe uma relação dicotômica entre esses distúrbios, pois os transtornos psiquiátricos em pacientes com abuso de substâncias tanto podem anteceder ao problema do uso abusivo de SPA, quanto ser uma consequência 
desse distúrbio (RIGGS, 2008).

Pacientes com concorrentes comorbidades e problemas com uso abusivo de substâncias são desafios especiais para o sistema de saúde em termos de diagnóstico e tratamento porque cada transtorno tem a capacidade de exacerbar o outro (RIGGS, 2008). Além disso, o tratamento desses distúrbios psiquiátricos que estão associados aos problemas relacionados ao uso de substâncias permite um melhor prognóstico nos resultados dos procedimentos (NARVAEZ, 2014) e a farmacoterapia tem papel fundamental nesse processo.

Transtornos Psicóticos: Os fármacos antipsicóticos atípicos têm sido alvo preferencial dos estudos com essa população em detrimento aos agentes antipsicóticos convencionais porque possuem menos efeitos colaterais que possam comprometer 0 cumprimento do tratamento, tratar de forma mais eficaz os sintomas negativos da esquizofrenia e diminuir a fissura pelas SPA.

A clozapina é o agente mais estudado nessa população, demonstrando num estudo comparativo com outros antipsicóticos, obter taxas de recaída menores (BRUNETTE, 2006). A olanzapina também se destaca em estudos por reduzir a fissura por cocaína e por obter mais tempo de descontinuação do uso de SPA, em comparação com risperidona e outros antipsicóticos típicos (SMELSON, 2006), além de ser considerada a molécula ideal para crise psicótica (VALERIANE, 2015). Outros fármacos foram testados para esta comorbidade específica com algum resultado positivo, como o aripiprazole para a fissura por álcool e cocaína e topiramato, dissulfiram e naltrexona na redução do uso de álcool (BRADY, 2007).

Transtornos de Humor: A comorbidade do tipo transtorno bipolar é considerada um potencial eixo causador de distúrbios por uso abusivo de drogas, uma vez que cerca de $50 \%$ dos indivíduos bipolares vai desenvolver algum tipo de problema por consumo de drogas durante a vida (BROWN, 2006). Testes comparando valproato de sódio mais de lítio com placebo mais lítio em indivíduos bipolares encontraram uma redução significativa na proporção de dias de consumo pesado, no grupo tratado com valproato. Notavelmente, a redução no consumo ocorreu na ausência de diferenças significativas de humor, sugerindo que o valproato pode ser utilizado nessa população (BRADY, 2007). A lamotrigina em monoterapia foi testada em indivíduos com transtorno bipolar e dependência de cocaína, resultando em melhorias no humor e diminuição do desejo pela droga (BROWN, 2006a).

Depressão: Embora a associação da depressão com abuso de substâncias esteja bem estabelecida, o assunto tem produzido debates quanto ao nexo de causalidade e a etiologia compartilhada, com os diagnósticos influenciando as decisões de tratamento para pacientes com ambos os transtornos.

Os resultados de estudos de tratamento antidepressivo em pacientes dependentes de substâncias com depressão têm sido altamente variáveis, onde a farmacoterapia antidepressiva tem obtido efeitos benéficos modestos quanto aos aspectos da dependência 
(BRADY, 2007).

Transtorno de Déficit de Atenção e Hiperatividade (TDAH): Dados epidemiológicos demonstram a importância da relação entre TDAH e distúrbios por uso abusivo de substâncias, pois cerca de $20 \%$ dos adultos com problemas pelo uso de drogas possuem esta comorbidade. Este fato coloca o TDAH como um fator de risco para o desenvolvimento em alguma dependência na idade adulta (WILENS e FUSILLO, 2007).

A intervenção farmacoterápica no tratamento é fundamental, pois quando os indivíduos são tratados em idades menores diminui efetivamente o risco para o consumo de cigarros e de ocorrência para distúrbios causados pelo consumo de drogas na idade adulta. De outra forma, o tratamento farmacológico utilizado sozinho em adultos com TDAH e distúrbios por uso de drogas se torna inadequado pelos resultados pouco promissores. $O$ fármaco mais utilizado para esta comorbidade é o metilfenidato, mas bupropiona, venlafaxina e atomoxetina também foram utilizados com resultados positivos (WILENS e FUSILLO, 2007).

Ansiedade: $O$ diagnóstico da ansiedade é dificultado quando se apresenta como uma comorbidade ao uso abusivo de drogas, pois muitos dos sintomas de abstinência de substâncias, principalmente na fase da desintoxicação, podem ser confundidos com os da ansiedade, podendo prejudicar sua identificação. Os antidepressivos tricíclicos têm recebido maior suporte empírico para o tratamento de transtornos de ansiedade, mas os seus perfis de efeitos colaterais e toxicidade impedem o seu uso como um tratamento de primeira linha, sendo os inibidores da recaptação da serotonina geralmente considerados como tal. Os benzodiazepínicos, classicamente ansiolíticos, não são recomendados para populações que fazem abuso de substâncias pelo potencial de dependência que apresentam (KELLY, 2012).

\section{FARMACOTERAPIA DAS SUBSTÂNCIAS DE ABUSO}

A farmacoterapia tem desempenhado um papel cada vez mais importante no tratamento da dependência, onde os medicamentos são utilizados principalmente como adjuvantes de tratamentos psicossociais. A atuação da farmacoterapia no tratamento do uso abusivo de SPA vai depender do tipo específico de droga e os fármacos podem ter como objetivos: gestão de síndromes de abstinência através da desintoxicação, atenuação do desejo em usar a droga (fissura), prevenção de recaída e redução de danos (DOUAIHY, 2013).

Nicotina (Tabaco): Embora os efeitos nocivos do tabagismo apareçam normalmente a longo prazo e os outros componentes do tabaco sejam os principais responsáveis, a nicotina é o agente causador da dependência. Os principais agentes farmacológicos que atuam nesse tipo de adição são:

- A bupropiona, um antidepressivo atípico com ações dopaminérgicas e adrenérgicas, e que atuaria como um antagonista no receptor nicotínico acetilcolinérgico. Seu 
efeito positivo na cessação em fumar parece ser independente da sua ação antidepressiva (HUGHES, 2007).

- A vareniclina, um agonista parcial do receptor da nicotina, que atua por combinação entre contrariar os sintomas de abstinência (atuando como um agonista) e reduzir a satisfação de fumar (agindo como um antagonista). Em estudos comparativos, teria ação superior a bupropiona na cessação do tabagismo (TONSTAD, 2010).

- A terapia de reposição de nicotina, que consiste em utilizar a nicotina em doses terapêuticas, sem outras toxinas presentes no cigarro, fornecendo um substituto parcial do hábito de fumar. A nicotina a partir do produto se liga a receptores nicotínicos acetilcolinérgicos do sistema nervoso central de uma forma dependente da dose, reduzindo o desejo de fumar e os sintomas de abstinência, bloqueando parcialmente o efeito de recompensa do cigarro (LINGFORD-HUGHES, 2012).

Álcool: A psicofarmacoterapia utilizada nos problemas causados pelo uso abusivo do álcool envolve os medicamentos para a desintoxicação, como os benzodiazepínicos, estabilização médica, tratamento da abstinência e dependência, com destaque para os três fármacos aprovados pelo FDA (food and drug administration) para este tipo de tratamento, dissulfiram, acamprosato e naltrexona, além do topiramato.

- Os benzodiazepínicos atuam sobre o GABA, diminuindo a atividade do sistema nervoso, auxiliando na desintoxicação do paciente através da redução da frequência cardíaca, pressão arterial, sudorese e ansiedade que estão associados ao processo de abstinência alcoólica. Além disso, previnem as convulsões e a ocorrência de delírio. Devem ser utilizados por curto prazo, pois geram tolerância e podem promover interações com pacientes que apresentarem recaídas (DOUAIHY, 2013).

- O dissulfiram é o primeiro medicamento aprovado pelo FDA para a dependência de álcool e está disponível há mais de 50 anos. Ele funciona inibindo a enzima aldeído desidrogenase, que converte o acetaldeído em acetato na quebra de álcool. 0 acúmulo de acetaldeído promove reações desagradáveis ao paciente, incluindo sudorese, náuseas, vômitos, rubor facial, taquicardia, cefaléia, falta de ar e hipotensão. Esse estado aversivo que serve para atenuar o comportamento dependente pelo reforço negativo, auxiliando a alcançar um período inicial de abstinência que melhoraria 0 envolvimento nos tratamentos psicossociais (POLYDOROU, 2008).

- A naltrexona é um medicamento para o tratamento de dependência de longo prazo, sendo um antagonista opioide competitivo que bloquearia os aspectos gratificantes de beber pela ocupação dos receptores opioides de beta-endorfinas. A diminuição a liberação de dopamina, reduziria o efeito inebriante do álcool. Além disso, uma hipótese para explicar a ação desse fármaco na redução em beber é que ele precipitaria a sedação, o que faria com que os pacientes evitem o álcool para atenuar a sedação adicional. (POLYDOROU, 2008).

- O acamprosato afeta diversos neurotransmissores e estruturalmente lembra GABA 
e glutamato. O glutamato é um dos principais neurotransmissores implicados no aumento da atividade neurológica e o acamprosato age sobre os receptores gabaérgicos, modulando o glutamato (POLYDOROU, 2008). Além disso, seu efeito antagonista do $\mathrm{N}$-metil-D-aspartato, explicaria seus efeitos positivos na diminuição da dependência. É mais eficaz quando administrado no período inicial após a cessação da abstinência aguda. Especificamente, o acamprosato age para diminuir a vontade de beber provocada pelo desejo de aliviar os sintomas de abstinência (DOUAIHY, 2013).

- O topiramato é um antiepilético que inibe as propriedades excitatórias da transmissão do glutamato. Acredita-se que esta propriedade pode diminuir a liberação de dopamina no nucleus accumbens em resposta ao consumo de álcool. Apesar do topiramato não ser aprovado pelo FDA para a dependência de álcool, dados comparando este anticonvulsivante com placebo mostraram uma redução no beber e aumento da abstinência (JOHNSON, 2007).

Maconha (Cannabis sativa): A dependência de cannabis está em terceiro lugar em termos de prevalência na população, atrás de que ao tabaco e álcool. Apesar disso, ainda não existe uma base clara de evidências para o tratamento farmacológico dessa adição e nenhuma farmacoterapia pode ser recomendada. As investigações se concentram principalmente no alívio dos sintomas de abstinência para ajudar nas tentativas de cessar o uso. Embora os sintomas se apresentem a nível emocional e comportamental, mudança de apetite, perda de peso e desconforto físico também são relatados (LINGFORDHUGHES, 2012).

- Testes realizados com tetrahidrocanabinol, lofexidina, rimonabant, carbonato de lítio, valproato de sódio, mirtazapina, fluoxetina e bupropiona foram realizados sem resultados positivos que pudessem indicar o seu uso no tratamento dessa dependência. (LINGFORD-HUGHES, 2012). A bupropiona inclusive piorou sintomas como irritabilidade, distúrbios do sono e alterações de humor (RIGGS, 2007).

Estimulantes (Cocaína, Anfetamina e derivados): As pesquisas em farmacoterapia para a dependência das substâncias estimulantes têm sido intensas e focam principalmente o tratamento de usuários dependentes de cocaína e anfetamina, onde os objetivos do tratamento geralmente são o manejo da retirada e a manutenção da abstinência, embora tratamentos de substituição com metas de redução de danos também sejam considerados. Apesar desses esforços, não existe uma farmacoterapia com evidência sólida o bastante para o tratamento dessa dependência.

- Agentes gabaérgicos (topiramato, tiagabina, baclofeno, vigabatrina), agonistas de reposição (modafinil, dissulfiram, metilfenidato), neurolépticos de segunda geração com ação em receptores serotoninérgicos (olanzapina e aripriprazol) e bupropiona (somente ela entre os agentes dopaminérgicos) demonstraram algum resultado positivo nos testes realizados, porém ainda sem comprovação de benefícios que resultassem em aprovação do seu uso para o tratamento da dependência dos 
estimulantes (SOFUOGLU, 2006; LINGFORD-HUGHES, 2012).

Opioides: As terapias farmacológicas mais eficazes para transtornos por uso de opioides são as terapias agonistas, onde os fármacos que ocupam os locais estimulados pelos opioides, possuem um perfil farmacocinético diferente (tempo de ação mais longo) e com menor efeito que as drogas de abuso, proporcionando menor reforço. A metadona e a buprenorfina são os agentes agonistas mais utilizados (DOUAIHY, 2013). Outro tipo de terapia utiliza fármacos como a naloxona e a naltrexona, com ação antagonista opioide, que diminui os efeitos prazerosos das SPA de abuso.

- A metadona é o medicamento mais estudado e utilizado nesse tipo de dependência, sendo aprovado pelo FDA para essa dependência. Também utilizado como analgésico da dor aguda (DOUAIHY, 2013). Tem ação agonista completa sobre os receptores opioides $\mu$ (mu) e tempo de meia vida de 24 horas (LINGFORD-HUGHES, 2012).

- A buprenorfina é um agente agonista opioide misto, capaz de produzir efeito agonista parcial nos receptores mu e efeito antagonista nos receptores $\mathrm{k}$ (kappa), resultando em bons resultados para o tratamento dessa dependência. Utilizada como analgésico potente, atua por até 48 horas e apresenta menores riscos de depressão respiratória que a metadona (DOUAIHY, 2013).

- A naloxona é um antagonista opioide usada com a buprenorfina para potencializar o efeito positivo no tratamento da dependência por opioides, sendo essa associação aprovada pelo FDA (DOUAIHY, 2013).

- Outro medicamento utilizado é a naltrexona, que atua como antagonista opioide que bloqueia os efeitos das substâncias de abuso. Deve ser utilizada após a cessação de uso dos opioides, pois pode ocasionar depressão respiratória em usuários ativos. Não possui potencial de abuso (LINGFORD-HUGHES, 2012).

Sedativos e Hipnóticos: Esta classe de substâncias de abuso tem como particularidade o fato de se apresentar normalmente como psicofármacos clássicos, onde o processo de dependência ocorre principalmente pelo tempo de uso prolongado, dose elevada e tempo de meia vida alto dos medicamentos (LADER, 2008).

Os medicamentos utilizados são os próprios benzodiazepínicos, agindo por serem agonistas, mas com tempo de meia vida maior, tendo redução gradual da dose e por pouco tempo de tratamento (LINGFORD-HUGHES, 2012). Outra opção é o uso do flumazenil, que é um antagonista competitivo e específico dos receptores benzodiazepínicos, revertendo seus efeitos. Deve ser utilizado com cuidado, pois pode ocasionar intoxicação por acúmulo de benzodiazepínicos (LADER, 2008).

\section{CONSIDERAÇÕES FINAIS}

A DQ é uma doença crônica que requer uma abordagem multiprofissional 
abrangente e de longo prazo, onde a compreensão dos mecanismos que determinam a ação das substâncias de abuso e dos próprios psicofármacos não é suficiente para a obtenção de resultados positivos do tratamento. Isto ocorre porque a DQ não depende apenas da substância de abuso, mas também outros fatores como aspectos individuais genéticos e coletivos sociais.

Os psicofármacos assumem importante função no processo por auxiliar os tratamentos psicossociais na desintoxicação, recuperação inicial e prevenção da recaída, mas seus benefícios são limitados. Apesar de apresentarem bons resultados no tratamento da dependência de álcool, nicotina e opioides, importantes adições como maconha, cocaína e seus derivados ainda não possuem tratamentos farmacológicos com evidências positivas comprovadas que permitam sua utilização.

Sendo assim, são necessárias mais pesquisas para a busca de novos medicamentos que auxiliem o tratamento dessas dependências, além da conscientização do paciente em participar ativamente do processo de recuperação, inclusive com ações não farmacológicas.

\section{REFERÊNCIAS}

BERRIDGE, K. C; ROBINSON, T. E; ALDRIDGE, J. W. Dissecting components of reward: liking, wanting, and learning. Curr Opin Pharmacology, n. 9, p. 65-73, 2009.

BRADY, K. T. et al. Treatment of Patients Comorbid for Addiction and Other Psychiatric Disorders. Current Psychiatry Reports, n.9, p. 374-80, 2007.

BROWN, E. S. et al. Naltrexone in patients with bipolar disorder and alcohol dependence. Depress Anxiety, n. 23, p. 492-95, 2006.

BROWN, E. S. et al. Lamotrigine for bipolar disorder and comorbid cocaine dependence: a replication and extension study. J Affect Disord, n. 93, p. 219-22, 2006.

BRUNETTE, M. F. et al.: Clozapine use and relapses of substance use disorder among patients with co-occurring schizophrenia and substance use disorders. Schizophr Bull, 32, p. 637-43, 2006.

DALLEY, J. W; EVERITT, B. J. Dopamine receptors in the learning, memory and drug reward circuitry. Semin Cell Dev Biol, n. 20, p. 403-10, 2009.

DEGENHARDT, L. et al. Global burden of disease attributable to illicit drug use and dependence: findings from the Global Burden of Disease Study 2010. Lancet, n. 382, p. 1564-74, 2013.

DOUAIHY, A. B; KELLY, T. M; SULLIVAN, C. Medications for Substance Use

Disorders. Social Work in Public Health, n. 28, p. 264-78, 2013.

FIGLIE, N. B; BORDIN, S; LARANJEIRA, R. Aconselhamento em dependência química. 2 ed. São Paulo, Ed Roca. 2010. 
FONSECA, V. A. S; LEMOS, T. Farmacologia na dependência química. In: DIHL, A; CORDEIRO, D. C; LARANJEIRA, R. Dependência química: prevenção, tratamento e políticas públicas. São Paulo, Ed. Artmed. p. 25-34, 2011.

FORRAY, A. SOFUOGLU, M . Future pharmacotherapies for substance use disorders. British Journal of Clinical Pharmacology, 77(2), p. 382-400, 2012.

FURST, S; RIBA, P; AL-KHRASANI, M. New approach to the neurobiological mechanisms of addiction. Neuropsychopharmacologia Hungarica, 15(4), p. 189-205, 2013.

HEIDBREDER, C. A; NEWMAN, A. H. Current perspectives on selective dopamine $D(3)$ receptor antagonists as pharmacotherapeutics for addictions and related disorders. Ann N Y Acad Sci, 1187, p. 4-34, 2010.

HORDER, J. et al. Reduced neural response to reward following 7 days treatment with the cannabinoid antagonist rimonabant in healthy volunteers. Int $\mathrm{J}$ Neuropsychopharmacol, n.13, p. 1103-13, 2010.

HUGHES, J. R; STEAD, L.F; LANCASTER, T. Antidepressants for smoking cessation. Cochrane Database of Systematic Reviews, 1. n. CD000031. DOI: 10.1002/14651858. CD000031.pub3, 2007.

JASINSKI, D. R; KRISHNAN, S. Abuse liability and safety of oral lisdexamfetamine dimesylate in individuals with a history of stimulant abuse. J Psychopharmacology, 9(23), p. 419-27, 2009.

JOHNSON, B. A. et al. Topiramate for treating alcohol dependence: a randomized controlled trial. JAMA, 298(14), p. 1641-51, 2007.

KELLY, T. M; DALEY, D. C; DOUAIHY, A. B. Treatment of substance abusing patients with comorbid psychiatric disorders. Addictive Behaviors, n. 37, p. 11-24, 2012.

KHOO, S. Y. S; BROWN, R. M. Orexin/Hypocretin Based Pharmacotherapies for the Treatment of Addiction: DORA or SORA? CNS Drugs, n. 28, p. 713-30, 2014.

KOOB, G. F; VOLKOW N. D. Neurocircuitry of addiction. Neuropsychopharmacology, n. 35, p. 217-38, 2010.

LADER, M. Effectiveness of benzodiazepines: do they work or not? Expert Rev Neurother, 8(8), p. 1189- 91, 2008.

LEVIN, F. R. et al. Treatment of cocaine dependent treatment seekers with adult ADHD: double-blind comparison of methylphenidate and placebo. Drug Alcohol Depend, n. 87, p. 20-9, 2007.

LINGFORD-HUGHES, A. R. et al. BAP updated guidelines: evidence-based guidelines for the pharmacological management of substance abuse, harmful use, addiction and comorbidity: recommendations from BAP. Journal of Psychopharmacology, 26(7), p. 899-952, 2012.

LONGO, M. et al. Randomized controlled trial of dexamphetamine maintenance for the treatment of methamphetamine dependence. Addiction, n.105, p. 146-54, 2010. 
MARTINEZ-RAGA, J; KNECHT, C; CEPEDA, S. Modafinil: a useful medication for cocaine addiction? Review of the evidence from neuropharmacological, experimental and clinical studies. Rev. Curr Drug Abuse, n.1, p. 213-21, 2008.

NARVAEZ, J. C. M. et al. Psychiatric and substance use comorbidities associated with lifetime crack cocaine use in young adults in the general population.

Comprehensive Psychiatry, n. 55, p. 1369-376, 2014.

PENG, X. Q. The preferential dopamine D3 receptor antagonist S33138 inhibits cocaine reward and cocaine triggered relapse to drug-seeking behavior in rats. Neuropharmacology, n. 56, p. 752-60, 2009.

POLYDOROU, P; LEVIN, F. R. Treating alcohol dependence: When and how to use 4 medications. Current Psychiatry, 7(2), p. 25-37, 2008.

RIGGS, P. D. et al. A randomized controlled trial of fluoxetine and cognitive behavioral therapy in adolescents with major depression, behavior problems, and substance use disorders. Arch Pediatr Adolesc Med, n. 161, p. 1026-34, 2007.

RIGGS, P. et al. Comorbid Psychiatrie and Substance Abuse Disorders: Recent Treatment Research. Substance Abuse, 29 (3), p. 51-63, 2008.

SCHMIDT, H. D; PIERCE, R. C. Cocaine induced neuroadaptations in glutamate transmission: potential therapeutic targets for craving and addiction. Ann N Y Acad Sci, 1187, p. 35-75, 2010.

SHOPTAW, S. Bupropion hydrochloride versus placebo, in combination with cognitive behavioral therapy, for the treatment of cocaine abuse/dependence. Journal Addict Dis, n. 27, p. 13-23, 2008.

SMELSON, D. A. et al. Antipsychotic treatment discontinuation among individuals with schizophrenia and co-occurring substance use. J Clin Psychopharmacology, n. 26, p. 666-67, 2006.

SOFUOGLU, M; KOSTEN, T. R. Emerging pharmacological strategies in the fight agaist cocaine addiction. Expert Opin Emerg Drugs, 11(1), p. 91-98, 2006.

TONSTAD, S; DAVIES, S; FLAMMER, M. et al. Psychiatric adverse events in randomized, double-blind, placebo-controlled clinical trials of varenicline: a pooled analysis. Drug Saf, n. 33, p. 289-301, 2010.

VALERIANE, G. et al. Olanzapine as the ideal "trip terminator"? Analysis of online reports relating to antipsychotics' use and misuse following occurrence of novel psychoactive substance-related psychotic symptoms Hum. Psychopharmacology Clin Exp. n. 30, p. 249-54, 2015.

VOLKOW, N. D; FOWLER, J. S; WANG, G. J. Positron emission tomography and single-photon emission computed tomography in substance abuse research. Semin. Nucl. Med, 33(2), p. 114-28, 2003.

UNITED NATIONS OFFICE ON DRUGS AND CRIME (UNODC). World Drug Report: executive summary. Viena, 2015. Disponível em: <http://www.unodc .org/documents 
/wdr2015/world_drug_report_2015.pdf> acesso em: 16 ago. 2016.

WILENS, T. E; FUSILLO, E. When ADHD and Substance Use Disorders Intersect:

Relationship and Treatment Implications. Current Psychiatry Reports, n. 9, p. 408-14, 2007. 